\title{
Photon transport in a dissipative chain of nonlinear cavities
}

\author{
Alberto Biella, ${ }^{1, *}$ Leonardo Mazza, ${ }^{1}$ Iacopo Carusotto, ${ }^{2}$ Davide Rossini, ${ }^{1}$ and Rosario Fazio ${ }^{1}$ \\ ${ }^{1}$ NEST, Scuola Normale Superiore \& Istituto Nanoscienze-CNR, I-56126 Pisa, Italy \\ ${ }^{2}$ INO-CNR BEC Center \& Dipartimento di Fisica, Università di Trento, I-38123 Povo, Italy
}

(Received 8 December 2014; published 12 May 2015)

\begin{abstract}
By means of numerical simulations and the input-output formalism, we study photon transport through a chain of coupled nonlinear optical cavities subject to uniform dissipation. Photons are injected from one end of the chain by means of a coherent source. The propagation through the array of cavities is sensitive to the interplay between the photon hopping strength and the local nonlinearity in each cavity. We characterize photon transport by studying the populations and the photon correlations as a function of the cavity position. When complemented with input-output theory, these quantities provide direct information about photon transmission through the system. The position of single-photon and multiphoton resonances directly reflects the structure of the many-body energy levels. This shows how a study of transport along a coupled cavity array can provide rich information about the strongly correlated (many-body) states of light even in presence of dissipation. The numerical algorithm we use, based on the time-evolving block decimation scheme adapted to mixed states, allows us to simulate large arrays (up to 60 cavities). The scaling of photon transmission with the number of cavities does depend on the structure of the many-body photon states inside the array.
\end{abstract}

DOI: 10.1103/PhysRevA.91.053815

\section{INTRODUCTION}

In the recent years, coupled cavity arrays (CCAs) [1-3] have been put forward as a very suitable playground for the investigation of quantum many-body phenomena in photonics systems. Due to their flexibility in the design, the possibility to control their dynamics (through the choice of the couplings and external drive) and the local accessibility of individual cavities, these systems have been proposed as possible implementations of a quantum simulator. A rather comprehensive account of the large body of work in this field can be found in Refs. [4-7]. The experimental requirements are quite challenging, however, in the last two years there have been very interesting progresses [8-10].

An important ingredient determining the dynamics of a cavity array is the competition between photon hopping and the nonlinearity present in each cavity, due to the coupling to a few-level system as for example in the Jaynes-Cummings model. Whereas tunneling between neighboring cavities tends to delocalize the photons, the presence of the nonlinearity, on the contrary, suppresses number fluctuations, thus opposing to delocalization. In the (hypothetical) absence of photon losses this competition would lead to a (thermo)dynamics similar to that of the Bose-Hubbard model. The properties of cavity arrays in this regime have been carefully scrutinized in the recent literature (see, e.g., the reviews [4-6]). The phase diagram in the one-dimensional case, related to this study, has been determined by means of density-matrix renormalization group in [11].

The presence of the unavoidable photon leakage would make the long-time dynamics completely trivial in the absence of an external drive that refills photons into the cavities. This additional competition, between losses and external pumping, makes the dynamical behavior of these systems particularly rich. The interplay of (coherent or incoherent) driving and (incoherent) photon losses can be both observed in the transient

*Corresponding author: alberto.biella@sns.it
PACS number(s): 42.50.Pq, 05.30.Jp, 37.10.Jk, 05.70.Ln

and in the steady-state (long-time) regime. In this work, we will be interested in the nonequilibrium steady state (NESS).

Only very recently, the many-body nonequilibrium dynamics of cavity arrays started to be addressed (see, e.g., Refs. [1220]) and several different properties both of the transient and steady-state regimes were highlighted. These include the spectroscopy of many-body photon states $[12,15-17,20]$, crystallization of photons [14], instabilities induced by pulsed driving [13], steady-state critical phenomena $[18,19]$. There is by now a compelling evidence that cavity arrays are naturally designed to become open-system quantum simulators.

Very interesting many-body effects should emerge in photon transport as well. Most of the attention so far has been devoted to the transmission of one or two photons. In this regime, transport in one-dimensional systems has been already studied in a variety of situations, in the presence of a single two-level system (see, for example, Refs. [21-24]), with extended nonlinearities [25] and in linear cavity arrays when the frequency of one or two cavities is tunable [26].

Moving away from the regime of few-photon transmission, it is natural to expect that the formation of many-body states of photons will considerably affect transport as well; to the best of our knowledge, this effect has not been investigated so far. It is important to stress from the beginning that it is not obvious how strong correlations play a role in photon transport. For a single cavity, the most striking effect is the photon blockade [27-30]. The presence of a single photon in a cavity, driven by an external coherent source, will block the passage of a second photon because of the strong nonlinearities present in the cavity itself. How is the photon blockade modified in an extended system? This is one of the questions that we will address.

We make a first step in this direction by addressing the problem of photon transport through a chain of nonlinear cavities in presence of dissipation. The setup we consider is sketched in Fig. 1. One end of the array is driven by a coherent laser source. We are interested in studying the light emerging at the other end of the array, linking its properties to 


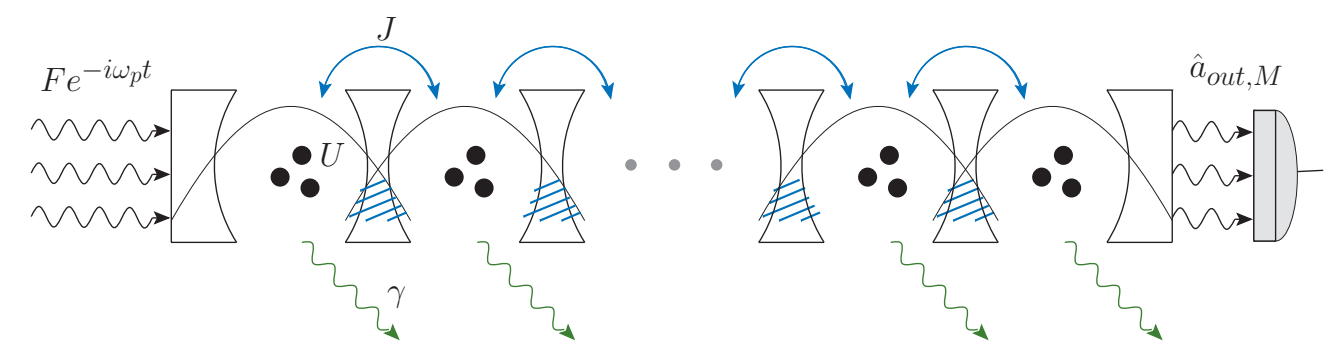

FIG. 1. (Color online) A sketch of the one-dimensional cavity array. Neighboring cavities are coupled by photon hopping. Nonlinearities in the cavities may produce an effective repulsion between the photons leading to an anharmonic spectrum. We consider a Kerr-type nonlinearity. Photons in the cavities have a finite lifetime therefore the cavities are pumped with an external coherent drive. Here, we suppose that only the leftmost cavity is pumped, in order to study photon transport through the system.

the presence of complex many-body states in the array itself. Our interest starts from Ref. [12], where it was shown that the steady state of a ring of nonlinear cavities, uniformly pumped, is reminiscent of a strongly correlated Tonks-Girardeau (TG) gas of impenetrable bosons. Inspired by this work, here we explore the impact of strongly correlated many-body states on transport.

Furthermore, it is worth mentioning at this stage that this system is also relevant for the understanding of single-photon sources based on passive photonics devices [31,32]. Changing a bit the perspective, the configuration we propose can be viewed as an alternative scheme to detect and quantify the presence of strongly correlated states of light based on transport. Usually, this kind of spectroscopic analysis is performed driving the whole array and studying the near-field and the far-field patterns.

In this work, we mainly focus in the regime where onsite nonlinearities are much stronger than photon hopping. First, we concentrate in the limit of impenetrable bosons: in this regime, repulsively interacting bosons form a Tonks-Girardeau (TG) gas and behave as noninteracting fermions [33]. Such limit is inaccessible with state-of-the-art experiments, but it is interesting in view of the rarity of exact solutions in many-body problems, and serves as a helpful benchmark for approximation methods in many-body theory.

Determining the steady state, i.e., the long-time limit of a many-body Lindblad equation, is a formidable task. In manybody open quantum systems the unavoidable exponential growth of the Hilbert space with the system size merges with the need to represent mixed states, leading to a huge number of degrees of freedom to be taken into account. By means of an extension of the time-dependent density-matrix renormalization group to open systems [34,35], we are able to attack this problem and study a large number (up to 60) of coupled cavities. Our numerical simulations are validated by means of an analytic approach based on effective models which take into account only few relevant degrees of freedom. The combination of (essentially) exact numerical methods together with the judicious construction of effective models allows us to considerably enrich the understanding of the underlying physics.

The paper is organized as follows. In the next section, we discuss in details the model for the driven and dissipative coupled cavity chain of Fig. 1. We will also discuss the basics of the input-output formalism that will allow us to compute transport properties. Sections III and IV are devoted to the presentation of our results; in Sec. III, we concentrate in the case of hard-core bosons, while the case of finite interaction strength is discussed in Sec. IV. Finally, in Sec. V we summarize our conclusions.

\section{MODEL}

We study transport properties of a one-dimensional array of $M$ optical cavities, coupled by photon tunneling, each one displaying an optical nonlinearity of the Kerr type. After tracing out the environmental degrees of freedom, the time evolution of the system density matrix is ruled by a master equation in the Lindblad form

$$
\dot{\rho}=-\frac{\mathrm{i}}{\hbar}[\hat{\mathcal{H}}, \rho]+\mathcal{L}[\rho],
$$

where the first term describes the coherent unitary time evolution, and the Lindblad term takes into account the damping. In the rest of the paper, we will consider independent photon losses from each cavity as the only dissipation channel.

Assuming that the spacing between the modes of each cavity is larger than any other involved energy scale, we can write the system Hamiltonian considering only one mode for each cavity

$$
\begin{aligned}
\hat{\mathcal{H}}_{0}= & \hbar \omega_{0} \sum_{i=1}^{M} \hat{a}_{i}^{\dagger} \hat{a}_{i}+\hbar U \sum_{i=1}^{M} \hat{a}_{i}^{\dagger} \hat{a}_{i}^{\dagger} \hat{a}_{i} \hat{a}_{i} \\
& -\hbar J \sum_{i=1}^{M-1}\left(\hat{a}_{i} \hat{a}_{i+1}^{\dagger}+\text { H.c. }\right),
\end{aligned}
$$

where $\hat{a}_{i}\left(\hat{a}_{i}^{\dagger}\right)$ are bosonic photon annihilation (creation) operators associated with the $i$ th cavity of the chain with resonance frequency $\omega_{0}$ which obey the canonical commutation relations $\left(\left[\hat{a}_{i}, \hat{a}_{j}^{\dagger}\right]=\delta_{i, j},\left[\hat{a}_{i}, \hat{a}_{j}\right]=0\right), J$ is the hopping rate and $U$ sets the scale of the Kerr nonlinearity.

The system is coherently driven by an incident (monochromatic) laser beam. In the setup we are interested in only the first cavity is coherently driven. In the input-output formalism [36], the equation of motion of the field operator in the first cavity (in the Heisenberg picture) is modified as follows:

$$
\partial_{t} \hat{a}_{1}(t)=\frac{\mathrm{i}}{\hbar}\left[\hat{\mathcal{H}}_{0}, \hat{a}_{1}(t)\right]-\frac{\kappa}{2} \hat{a}_{1}(t)+\sqrt{\kappa} \hat{a}_{\text {in, } 1}(t),
$$

where $\hat{a}_{\text {in, } 1}$ is the (laser) input field shined on the first cavity and $\kappa$ is the coupling between the cavity mode $\hat{a}_{1}$ and the laser 
field. The laser is in a coherent state and then can be written as the input relative to the vacuum $\hat{a}_{\mathrm{in}, 1}^{\mathrm{vac}}$ displaced by $\alpha$ and rotating at the pump frequency $\omega_{p}\left(\hat{a}_{\mathrm{in}, 1}=\hat{a}_{\mathrm{in}, 1}^{\mathrm{vac}}+\alpha e^{-\mathrm{i} \omega_{p} t-\mathrm{i} \phi}\right)$. By substituting it in Eq. (3) we get

$$
\begin{aligned}
\partial_{t} \hat{a}_{1}(t)= & \frac{\mathrm{i}}{\hbar}\left[\hat{\mathcal{H}}_{0}, \hat{a}_{1}(t)\right]-\frac{\kappa}{2} \hat{a}_{1}(t) \\
& +\sqrt{\kappa}\left[\hat{a}_{\mathrm{in}, 1}^{\mathrm{vac}}(t)+\alpha e^{-i \omega_{p} t-\mathrm{i} \phi}\right] .
\end{aligned}
$$

The last term on the right-hand side of Eq. (4) can be taken into account at the Hamiltonian level by adding a term to $\hat{\mathcal{H}}_{0}$,

$$
\hat{\mathcal{H}}=\hat{\mathcal{H}}_{0}+\hbar\left[F(t) \hat{a}_{1}^{\dagger}+F^{*}(t) \hat{a}_{1}\right],
$$

where $F(t)=F e^{-i \omega_{p} t}$ with $F=|F(t)|=\sqrt{\kappa} \alpha$ and $\phi=\pi / 2$. After transforming to a frame rotating with the laser frequency $\omega_{p}$, the Hamiltonian takes the form

$$
\hat{\mathcal{H}}=\hat{\mathcal{H}}_{0}-\hbar \omega_{p} \sum_{i=1}^{M} \hat{a}_{i}^{\dagger} \hat{a}_{i}+\hbar F\left(\hat{a}_{1}^{\dagger}+\hat{a}_{1}\right) .
$$

The second and third terms in Eq. (4) take into account the noise induced from this input-output channel and can be safely neglected because the main noise source is due to the uniform photon loss at rate $\gamma$, that is uniform for all the cavities $(\kappa / \gamma \ll$ 1). The corresponding Lindblad term reads as

$$
\mathcal{L}[\rho]=\frac{\gamma}{2} \sum_{i=1}^{M}\left(2 \hat{a}_{i} \rho \hat{a}_{i}^{\dagger}-\hat{a}_{i}^{\dagger} \hat{a}_{i} \rho-\rho \hat{a}_{i}^{\dagger} \hat{a}_{i}\right) .
$$

The nontrivial competition between unitary time evolution and Lindblad dissipation leads to NESS given by the stationary point of the above master equation $(\dot{\rho}=0)$. Specifically, we analyze the population and the statistics of the light transmitted by the array $\hat{a}_{\text {out }, M}$. Again employing input-output theory one can relate the behavior of $\hat{a}_{\text {out }, M}$ to the field in the last cavity of the array $\hat{a}_{M}$. For example, using the relation $\hat{a}_{\text {out }, M}=\hat{a}_{\text {in }, M}+$ $\sqrt{\kappa^{\prime}} \hat{a}_{M}$ and exploiting the fact that $\hat{a}_{\mathrm{in}, M}$ is just the vacuum, for the population one gets

$$
\left\langle\hat{a}_{\text {out }, M}^{\dagger} \hat{a}_{\text {out }, M}\right\rangle=\kappa^{\prime}\left\langle\hat{a}_{M}^{\dagger} \hat{a}_{M}\right\rangle,
$$

where $\kappa^{\prime}$ takes into account the coupling of the last cavity to the outside. The notation $\langle\hat{O}\rangle$ indicates the expectation value of the operator $\hat{O}$ taken in the standard way $\langle\hat{O}\rangle=\mathcal{Z}^{-1} \operatorname{Tr}\left[\rho^{\text {NESS }} \hat{O}\right]$, where $\mathcal{Z}=\operatorname{Tr} \rho^{\text {NESS }}$ is the partition function and $\rho^{\text {NESS }}$ is the NESS density matrix. Remarkably, the photon statistic will also be exactly the same as the cavity field [36]. For this reason, in this work we show results about the photon density and the correlations of the field in the $M$ th cavity. As we did previously for the first cavity, we neglect the noise contribution coming from this input-output channel. However, it would be not a problem to rigorously include such noise terms in our model by modifying the loss rate of the first and $M$ th cavity. In the following, we will fix $\hbar=1$ and work in units of $\gamma$.

As mentioned before, from a computational point of view, the simulation of Eq. (1) brings together both the complexity due to the exponential growth of the Hilbert space with the system size and the mixed-states dynamics generated by the nonunitary time evolution. Since we are interested in describing large arrays, in order to overcome this issue we exploit an algorithm based on the time-evolving block

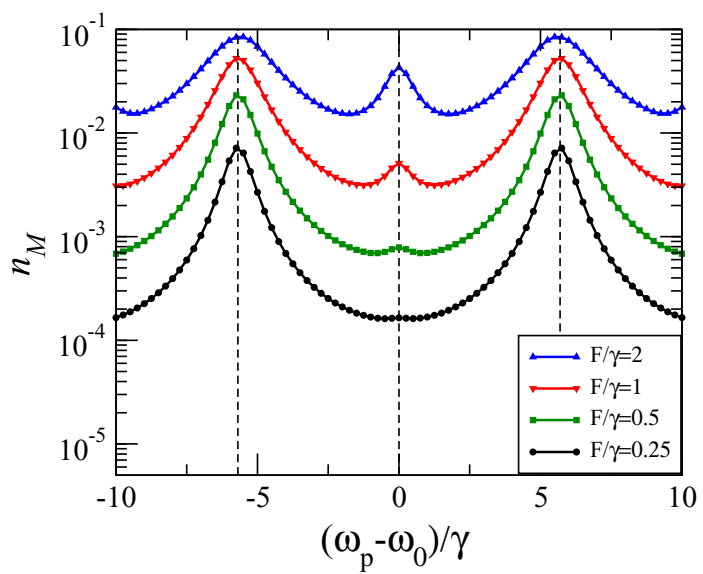

FIG. 2. (Color online) The population in the $M$ th cavity $n_{M}$ in the NESS as a function of the detuning $\left(\omega_{p}-\omega_{0}\right) / \gamma$ for different values of the driving strength. The dashed vertical lines are the spectral positions of the peaks in the fermionized limit $\left(\left|k_{5}\right\rangle,\left|k_{5}, k_{6}\right\rangle\right.$, and $\left|k_{6}\right\rangle$ from left to right). The parameters are $U / J=\infty, J / \gamma=20$, $\omega_{0} / \gamma=1$, and $M=10$.

decimation (TEBD) scheme [37,38] extended to open systems $[34,35]$. This relies on the representation of the density matrix as a matrix product operator (MPO) and can be viewed as a generalization of a matrix product state (MPS) for nonpure states. In this work, we simulate chains with a number of cavities up to $M=60$. The bond-link dimension used is $\chi=100$. In our system, for typical values of parameter, this representation allows us to capture most of the entanglement in the NESS. In Appendix B, we recall the basic features of the algorithm in order to give immediate meaning to the quantities introduced to obtain accurate numerical simulations.

In order to gain further insight, we will supply the MPO simulation with some effective models which are able to capture the main features of the NESS. The structure of such effective models is detailed in Appendix A.

\section{TRANSPORT IN THE TONKS-GIRARDEAU LIMIT}

Let us first consider the limit of impenetrable bosons $(U / J=+\infty)$ : in this regime, repulsively interacting bosons form a TG gas and behave as noninteracting fermions [33]. In Fig. 2, the population in the $M$ th cavity $n_{M}=\left\langle\hat{a}_{M}^{\dagger} \hat{a}_{M}\right\rangle$ is shown as a function of the pump frequency $\omega_{p}$. Looking at Fig. 2, in the detuning range shown, we note the presence of two main peaks (symmetrically displaced w.r.t. the zero detuning point) for all the values of the driving strength probed and a peak at zero detuning which emerges as the driving strength is increased. In order to understand the nature of this peak, it is necessary to analyze the many-body spectrum of $\hat{\mathcal{H}}_{0}$.

As shown by Girardeau in Ref. [33], the generic $N$-particle eigenstate of the closed system $\hat{\mathcal{H}}_{0}$ can be exactly mapped into a fermionic one. For $N$ bosons, the wave function in real-space representation is given by

$$
\Psi_{B}\left(i_{1}, \ldots, i_{N}\right)=\prod_{k<j}^{N} \operatorname{sgn}\left(i_{k}-i_{j}\right) \Psi_{F}\left(i_{1}, \ldots, i_{N}\right) .
$$


The term $\prod_{k<j}^{N} \operatorname{sgn}\left(i_{k}-i_{j}\right)$ ensures that $\Psi_{B}\left(i_{1}, \ldots, i_{N}\right)$ is symmetric under the exchange of any two particles. In this limit, the eigenstates of $\hat{\mathcal{H}}_{0}$ can be simply labeled by the occupation number of the single-particle eigenstates of the noninteracting Hamiltonian, with the prescription that we cannot put more than one particle in each orbital. The notation $\left|k^{(1)}, \ldots, k^{(N)}\right\rangle$ indicates the $N$-boson wave function, obtained by the symmetrization of the fermionic one via Eq. (9), with one particle in each $k^{(1)}, \ldots, k^{(N)}$ orbital. The energy of the $N$-boson wave function $\left|k^{(1)}, \ldots, k^{(N)}\right\rangle$ is identical to the corresponding fermionic one, i.e., $E=\sum_{\alpha=1}^{N} \mathcal{E}\left(k^{(\alpha)}\right)$ where $\mathcal{E}\left(k^{(\alpha)}\right)=\omega_{0}-2 J \cos k^{(\alpha)}$ and the momenta $k^{(\alpha=1, \ldots, N)}$ are to be chosen in the set $k_{n}=n \pi /(M+1)$ with $n=1, \ldots, M$, as imposed by the open boundary conditions.

The peaks in the transmission spectrum are due to the fact that the laser frequency is resonant with some eigenstates of $\hat{\mathcal{H}}_{0}$. Transport, in this regime, occurs through extended manybody photon states of the global system. This is the manybody extension of the classic photon-blockade effect in the single driven cavity. The extended many-body states govern the transport in all the cases we considered up to the largest chains of about 60 cavities. It is not obvious that this should be the case since incoherent photon leakage occurs in each cavity while driving is only through the first cavity only. Here, and in the rest of the paper, we demonstrate that photon transport can be dominated by extended many-body effects.

Figure 2 shows that, when the driving strength is weak $(F / \gamma \ll 1)$, only the one-photon states (in the range shown, $\left|k_{5}\right\rangle$ and $\left.\left|k_{6}\right\rangle\right)$ are excited by the pump. Remarkably, in this driving scheme, all the one-particle states are coupled to the vacuum with a matrix element $F_{k_{n}}=\left\langle k_{n}|\hat{\mathcal{H}}| 0\right\rangle=$ $F \sqrt{2 /(M+1)} \sin k_{n}$. Increasing the driving strength, manybody states start to be excited, due to the sequential absorption of $N$ photons from the drive. This means that a peak in the spectrum relative to the many-body state $\left|k^{(1)}, \ldots, k^{(N)}\right\rangle$ will appear at $\omega_{p}=\sum_{\alpha=1}^{N} \mathcal{E}\left(k^{(\alpha)}\right) / N$. Specifically, in Fig. 2, the two-photon resonance relative to the state $\left|k_{5}, k_{6}\right\rangle$ appears at $\omega_{p}=\left[\mathcal{E}\left(k_{5}\right)+\mathcal{E}\left(k_{6}\right)\right] / 2$ as the strength of the pump is increased. As it is typical in a driven-dissipative scenario, driving and losses imply transitions between eigenstates of $\hat{\mathcal{H}}_{0}$ with different number of particles and are responsible of the finite linewidth of the resonances. Such broadening increases as the driving strength is increased and, as a result, the background due to the off-resonant excitation of the eigenstates of $\hat{\mathcal{H}}_{0}$ becomes more and more important.

\section{A. One-photon resonances}

Starting from these initial observations, we want to investigate the structure of the NESS when the driving laser is resonant with a one-photon state

$$
\left|k_{n}\right\rangle=\sqrt{\frac{2}{M+1}} \sum_{i=1}^{M} \sin \left(k_{n} i\right) \hat{a}_{i}^{\dagger}|0\rangle .
$$

In Fig. 3, the typical behavior of the population in the $M$ th cavity as a function of the driving strength is shown.

We compare the numerical data (symbols) with the outcome of a truncated effective model (solid lines) which involves only the one-body states $\left|k_{m}\right\rangle$ and the vacuum $|0\rangle$. In this way, we

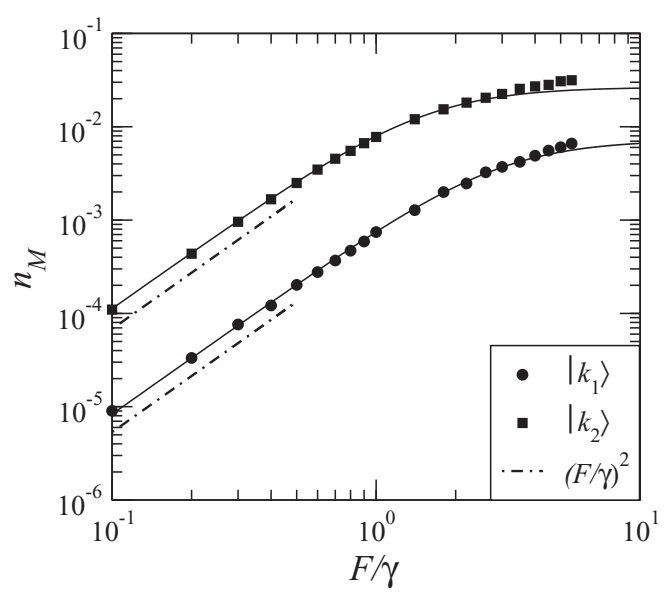

FIG. 3. The population in the $M$ th cavity $n_{M}$ in the NESS as a function of the pump amplitude $F / \gamma$, when the one-photon resonance condition is satisfied for the states $\left|k_{1}\right\rangle$ and $\left|k_{2}\right\rangle$. Symbols denote the numerical data, while solid lines are the outcomes of the OBM (or equivalently of the TLM). Dashed lines indicate a behavior $n_{T} \propto$ $(F / \gamma)^{2}$, and are plotted to guide the eye. The parameters are the same as in Fig. 2.

take into account both the resonant state $\left|k_{n}\right\rangle$ and the remaining off-resonant one-body states. In what follows, we will refer to this model as the one-body model (OBM). The numerics perfectly agrees with the data of the OBM for $F / \gamma \ll 1$, where $n_{M} \propto(F / \gamma)^{2}$. For $F / \gamma \gg 1$, the OBM underestimates the population because the off-resonant excitation of many-body states starts to be relevant.

If the one-photon resonances are well separated in energy with respect to their width, one can further simplify the model considering the resonant state $\left|k_{n}\right\rangle$ and the vacuum only $|0\rangle$, so that an effective two-level model is obtained and can be analytically solved (see Appendix A). We will refer to this model as the two-level model (TLM). This is the case, for example, of the data in Fig. 3, where the population in the $M$ th cavity in the NESS is shown as a function of the driving strength for an array of 10 cavities. In this case, the discrepancy between the predictions of the OBM and the TLM is not appreciable.

In Fig. 4, the typical density profile on resonance with a one-photon state $\left|k_{n}\right\rangle$ is shown. As explained above, for small system sizes (top panels) the one-photon resonances are well separated in energy compared to their width and then the contribution to the NESS of the off-resonant states is strongly suppressed. As a result, the NESS is a mixture of $\left|k_{n}\right\rangle$ and $|0\rangle$ only, and the density profiles clearly have a sinusoidal shape with wave vector $k_{n}$. Nevertheless, small differences between the OBM (solid lines) and the TLM (dashed lines) are visible for the resonance relative to the state $\left|k_{1}\right\rangle$. As the system size is increased (middle and bottom panels), the occupation of the off-resonant one-photon states is not negligible, thus resulting in a more complicated structure of the NESS. It is important to note that, in the parameter range spanned here and for the considered system sizes, we do not observe an exponential suppression of the photon density. Nonetheless, such exponential behavior is expected for large sizes (where the resonance peaks can no longer be resolved in the spectrum), 

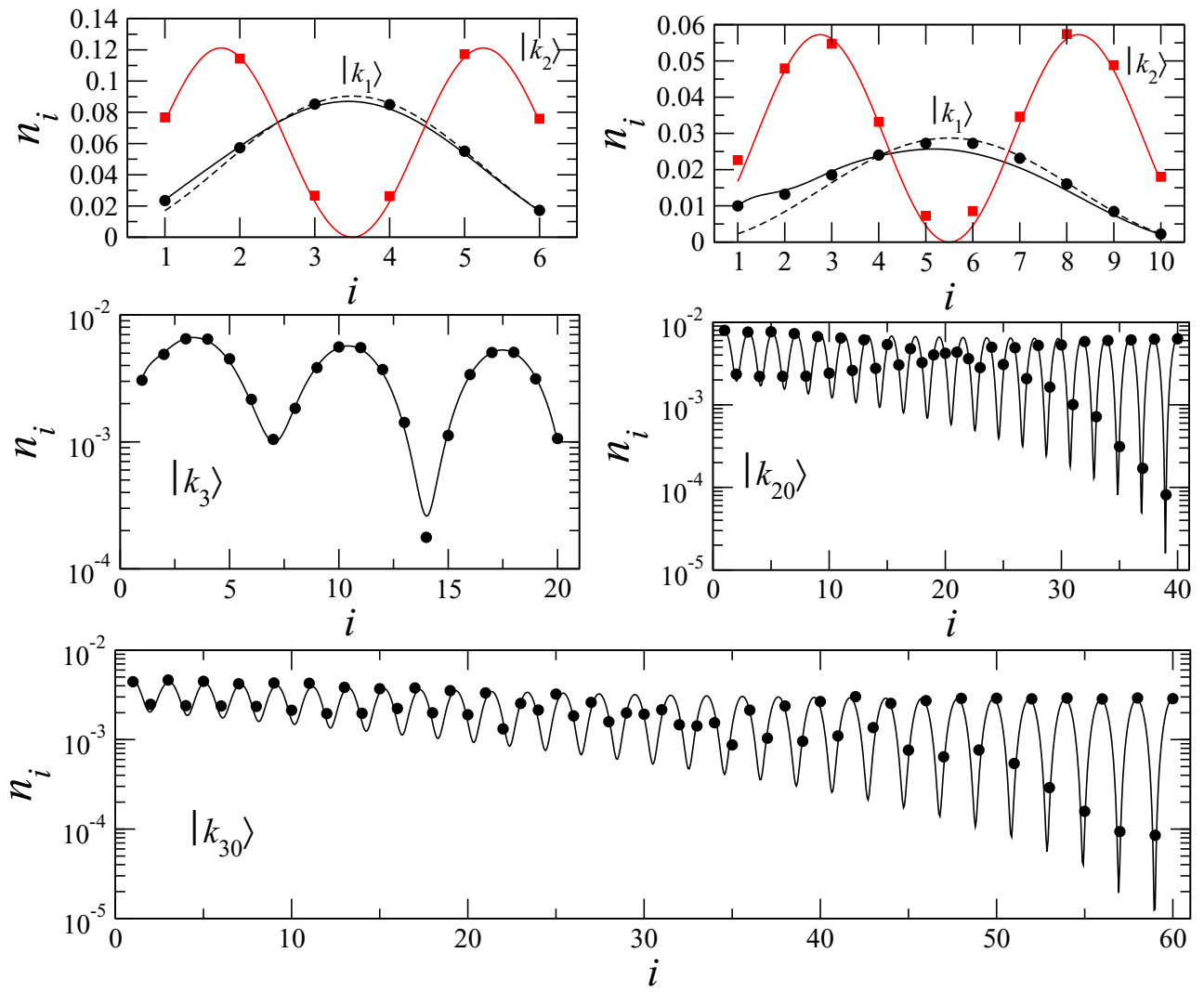

FIG. 4. (Color online) Photon occupations of each site in the NESS, when the one-photon resonance condition is satisfied for different states (as indicated in each panel) and for different system sizes (respectively, $M=6,10,20,40,60$ ). Symbols denote the numerical data, solid lines are the outcomes of the OBM, and dashed lines are the results of the TLM (shown for $M=6,10$ ). The parameters are the same as in Fig. 2, but with $F / \gamma=2$ for $M=6,10$ and $F / \gamma=1$ in the other panels.

a situation in which the system can be understood as an optical medium supporting a continuum of modes obeying a certain dispersion relation. Given that the wave propagation through the medium is affected by photon leakage that occurs from each of the cavities, the photon density is expected to be exponentially suppressed at a rate which depends on the laser frequency.

\section{B. Many-photon resonances}

More interesting is the characterization of the NESS in correspondence of the $N$-photon peaks. In this case, the NESS will be a mixture of one-photon states and many-body states. Our aim is to quantify the presence of many-body states in the NESS and to study their signatures on the observables. We start analyzing the excitation of a generic two-photon state $\left|k_{p}, k_{q}\right\rangle$. Considering Eq. (9), the generic two-photon eigenstate of $\hat{\mathcal{H}}_{0}$ can be written as

$$
\left|k_{p}, k_{q}\right\rangle=\frac{1}{M+1} \sum_{i, j=1}^{M} f_{k_{p}, k_{q}}(i, j) \hat{a}_{i}^{\dagger} \hat{a}_{j}^{\dagger}|0\rangle,
$$

with $\quad f_{k_{p}, k_{q}}(i, j)=\operatorname{sgn}(i-j)\left[\sin \left(k_{p} i\right) \sin \left(k_{q} j\right)-\right.$ $\left.\sin \left(k_{p} j\right) \sin \left(k_{q} i\right)\right]$. When the two-photon resonance condition is satisfied,

$$
\omega_{p}=\frac{1}{2}\left[\mathcal{E}\left(k_{p}\right)+\mathcal{E}\left(k_{q}\right)\right],
$$

the two-body state $\left|k_{p}, k_{q}\right\rangle$ cannot be directly excited by the driving. The occupation of $\left|k_{p}, k_{q}\right\rangle$ is the result of the sequential absorption of two photons by the off-resonance one-body states $\left|k_{p}\right\rangle$ and $\left|k_{q}\right\rangle$. In fact, the one-photon states $\left|k_{n}\right\rangle$ are coupled to the two-photon states $\left|k_{p}, k_{q}\right\rangle$ with a

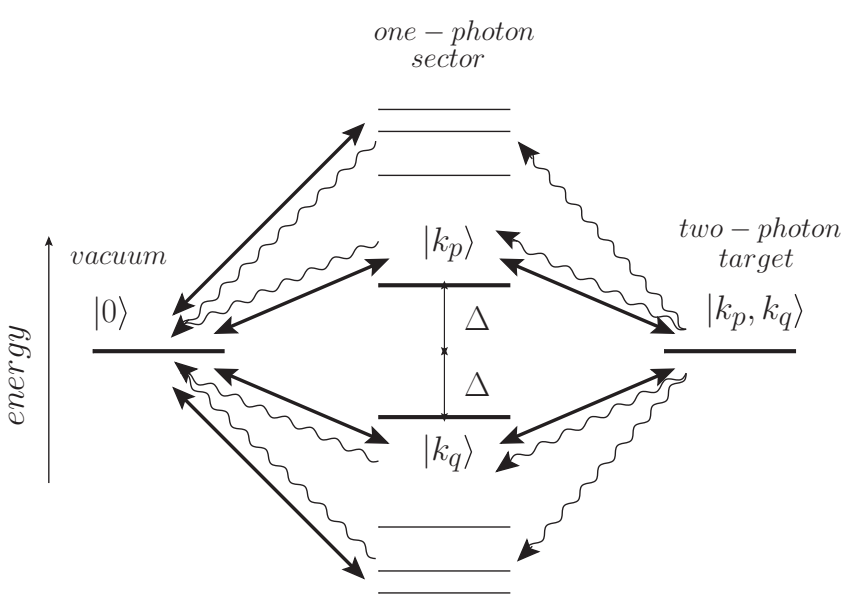

FIG. 5. Typical level scheme on resonance with a two-body state $\left|k_{p}, k_{q}\right\rangle$. The solid arrows stand for coherent Hamiltonian couplings, while wavy arrows indicate the decay induced by the Lindbladian. 

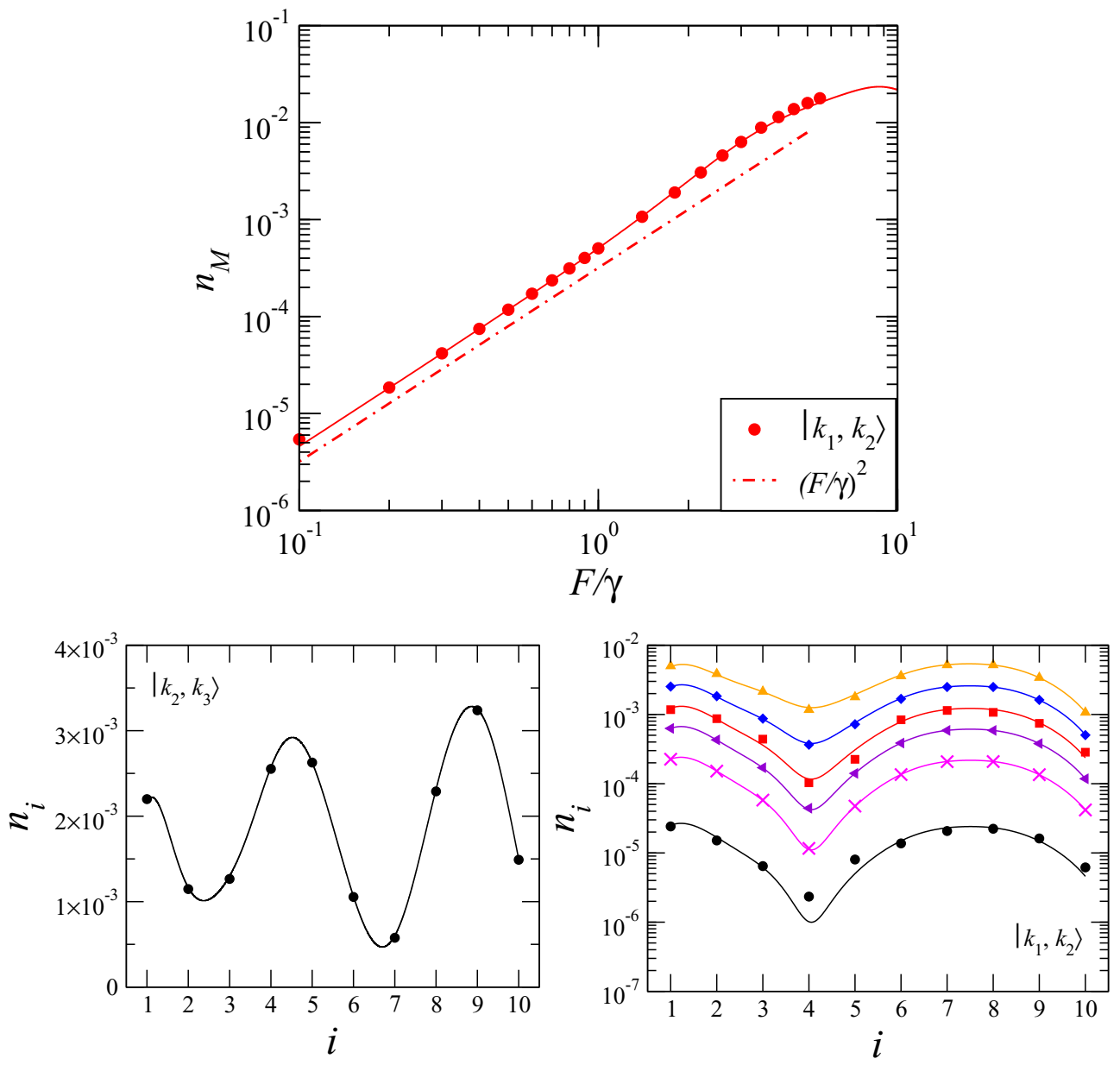

FIG. 6. (Color online) Top panel: the population in the $M$ th cavity as a function of the pump amplitude, when the two-photon resonance condition (12) is satisfied. Symbols denote the numerical data, while solid lines are the TBM predictions. The dashed line indicates a behavior $n_{T} \propto(F / \gamma)^{2}$, and is plotted to guide the eye. The parameters are the same as in Fig. 2. Bottom panels: photon occupations of each site in the NESS, when the two-photon resonance condition (12) is satisfied. Symbols are the numerical data, while solid lines are the TBM predictions. Left panel: $F / \gamma=1$ and the target state is $\left|k_{2}, k_{3}\right\rangle$. Right panel: $F / \gamma=0.1,0.3,0.5,0.7,1,1.4$ (from bottom to top, respectively) and the target state is $\left|k_{1}, k_{2}\right\rangle$. In both panels, the remaining parameters are set as in Fig. 2.

matrix element

$$
\begin{aligned}
A_{k_{n}, k_{p}, k_{q}} & =\left\langle k_{n}|\hat{\mathcal{H}}| k_{p}, k_{q}\right\rangle \\
& =F \sqrt{\frac{2}{M+1}}\left(\delta_{k_{n}, k_{p}} \sin k_{q}-\delta_{k_{n}, k_{q}} \sin k_{p}\right) .
\end{aligned}
$$

It is interesting to note that despite the rich structure of the two-photon state $\left|k_{p}, k_{q}\right\rangle$ in momentum space, shining only the first cavity (or equivalently the last one) implies that $\left|k_{p}, k_{q}\right\rangle$ is directly (coherently) coupled only to the states $\left|k_{p}\right\rangle$ and $\left|k_{q}\right\rangle$ (see Appendix A). On the other hand, the Lindbladian terms in the master equation (1) allow (incoherent) transition from $\left|k_{p}, k_{q}\right\rangle$ to different one-body states. The situation is depicted in Fig. 5.

In the top panel of Fig. 6, the population in the $M$ th cavity is plotted as a function of the driving strength. The numerics is compared with the results of an effective model which involves all the one-body states and the target two-photon state $\left|k_{p}, k_{q}\right\rangle$. We will refer to this model as the two-body model (TBM). As expected, for weak driving strength, the contribution to the population in the $M$ th cavity is almost completely given by the sum of the populations of the single-particle states, and then $n_{M} \propto(F / \gamma)^{2}$. For $F / \gamma>1$, the population in the $\left|k_{p}, k_{q}\right\rangle$ state becomes not negligible, thus resulting in a more complex behavior of which deviates from $n_{M} \propto(F / \gamma)^{2}$. We note that our theoretical predictions are in good agreement with the numerical data for all the probed values of $F / \gamma$. However, for $F / \gamma \gg 1$, any kind of effective model is expected to fail because of the not negligible excitation of the states not included in the model. Also, the local density in the NESS (see bottom panels of Fig. 6) is captured by the TBM.

In order to disclose information about the correlations in the NESS, we studied the normalized two-body function

$$
g^{(2)}(i, j)=\frac{\left\langle\hat{a}_{i}^{\dagger} \hat{a}_{j}^{\dagger} \hat{a}_{i} \hat{a}_{j}\right\rangle}{\left\langle\hat{a}_{i}^{\dagger} \hat{a}_{i}\right\rangle\left\langle\hat{a}_{j}^{\dagger} \hat{a}_{j}\right\rangle} .
$$

Such quantity is directly deducible from photocorrelation signals measurement. Furthermore, being the $g^{(2)}$ a statistical normalized quantity, this is of particular interest in the case of weak laser strength, when the number of photons in the array is very small. Supposing that the only populated two-body state 

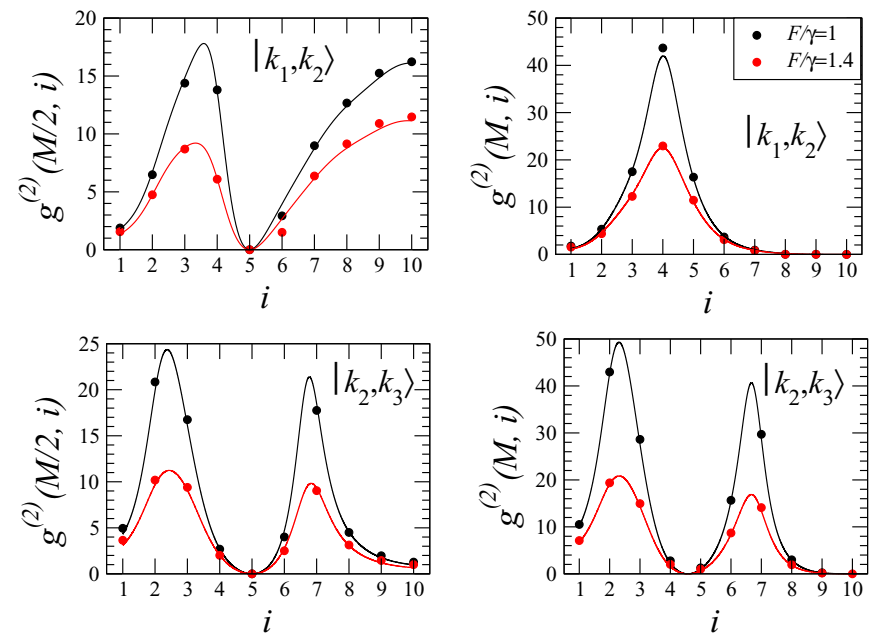

FIG. 7. (Color online) Normalized two-body correlation function when the two-photon resonance condition (12) is satisfied. Symbols denote the numerical data, while solid lines are the results of the TBM. The data are taken for two values of $F / \gamma$, as indicated in the legend. Top panels refer to the target state $\left|k_{1}, k_{2}\right\rangle$, while the lower ones to $\left|k_{2}, k_{3}\right\rangle$. The remaining parameters are set as in Fig. 2. On the left plots the $g^{(2)}$ function is plotted starting from the center of the array, while on the right plot we considered the $M$ th cavity as reference.

is $\left|k_{p}, k_{q}\right\rangle$, the normalized two-body function reads as

$$
g^{(2)}(i, j)=\frac{\rho_{2 p h}^{\mathrm{NESS}}}{n_{i} n_{j}}\left(\frac{2}{M+1}\right)^{2} f_{k_{p}, k_{q}}^{2}(i, j)
$$

where $\rho_{2 p h}^{\mathrm{NESS}}=\left\langle k_{p}, k_{q}\left|\rho^{\mathrm{NESS}}\right| k_{p}, k_{q}\right\rangle$ is the population of the target two-body state in the NESS and $n_{i(j)}$ are the local densities. As is clear from Eq. (15), the two-body function is directly related to the correlations in the target state.

The results are shown in Fig. 7 for different target states and for different values of $F / \gamma$. Specifically, when the two-body correlation function is plotted taking as reference the center of the array (left panels), the autocorrelation displays perfect antibunching $g^{(2)}(M / 2, M / 2)=0$ as expected in TG limit, while the cross correlations show an oscillatory behavior well captured by the TBM. In the spirit of a transport setup, we also considered as a reference the end of the array (right panels). Also in this case the autocorrelation of the light in the last cavity reveals a perfectly antibunched statistics $g^{(2)}(M, M)=$ 0 , and the numerical data are in good agreement with those of the effective model.

\section{TRANSPORT AT FINITE INTERACTION}

We now turn to the situation where the onsite nonlinearity is finite $(U / J<\infty)$. For this purpose, it is instructive to start from the noninteracting case $(U / J=0)$, which is discussed in Sec. IV A. This will be helpful to understand the physics at finite interactions, as analyzed in detail in Sec. IV B, and its relation with the findings in the TG limit.
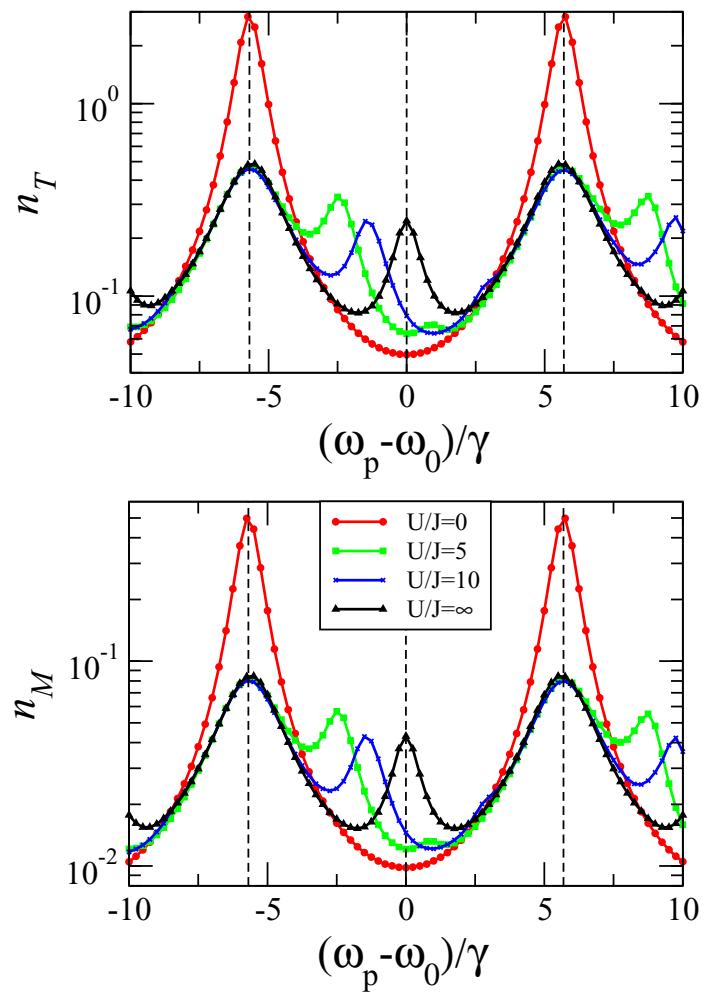

FIG. 8. (Color online) Total population (top panel) and population in the $M$ th cavity (bottom panel) as a function of laser detuning. The various symbols stand for different values of $U / J$, as indicated in the legend. The dashed vertical lines are $\left|k_{5}\right\rangle,\left|k_{5}, k_{6}\right\rangle$, and $\left|k_{6}\right\rangle$, respectively. In both panels $F / \gamma=2, J / \gamma=20, \omega_{0} / \gamma=1$, and $M=10$.

\section{A. Linear case}

The case of a linear chain of cavities is integrable because both the Hamiltonian and the Lindbladian are quadratic in the photon creation and annihilation operators. The equations of motion of the needed observables are identical to the corresponding classical equations (see for example Ref. [7]) and can be solved for the NESS using the fourth-order Runge-Kutta method. The total population in the NESS and the population in the $M$ th cavity of the chain are displayed in Fig. 8 as a function of laser detuning. In the range shown, the visible single-particle states (whose energy does not depend on $U / J)$ are $\left|k_{5}\right\rangle$ and $\left|k_{6}\right\rangle$. In the free case, the states $\left|k_{n}\right\rangle$, $\left|2: k_{n}\right\rangle,\left|3: k_{n}\right\rangle, \ldots$ (the notation $\left|N: k_{n}\right\rangle$ indicates the state with $N$ particles in the $k_{n}$ orbital) can be all resonantly excited (dependently on the driving strength $F / \gamma$ ) at $\omega_{p}=\mathcal{E}\left(k_{n}\right)$. This means that, on resonance, the NESS will be a mixture of one-body and (factorizable) many-body states composed of photons with the same momenta: the photon blockade is absent because of the harmonicity of the resulting spectrum.

It is important to note that the excitation of many-body states composed by photons with different momenta is strongly suppressed because of a destructive interference phenomena in the excitation pathways. The two main processes involved in the excitation of the generic two-photon state $\left|1: k_{p} ; 1: k_{q}\right\rangle$ with $k_{p} \neq k_{q}$ are $|0\rangle \rightarrow\left|k_{p}\right\rangle \rightarrow\left|1: k_{p} ; 1: k_{q}\right\rangle$ and $|0\rangle \rightarrow\left|k_{q}\right\rangle \rightarrow\left|1: k_{p} ; 1: k_{q}\right\rangle$. These can occur with the 


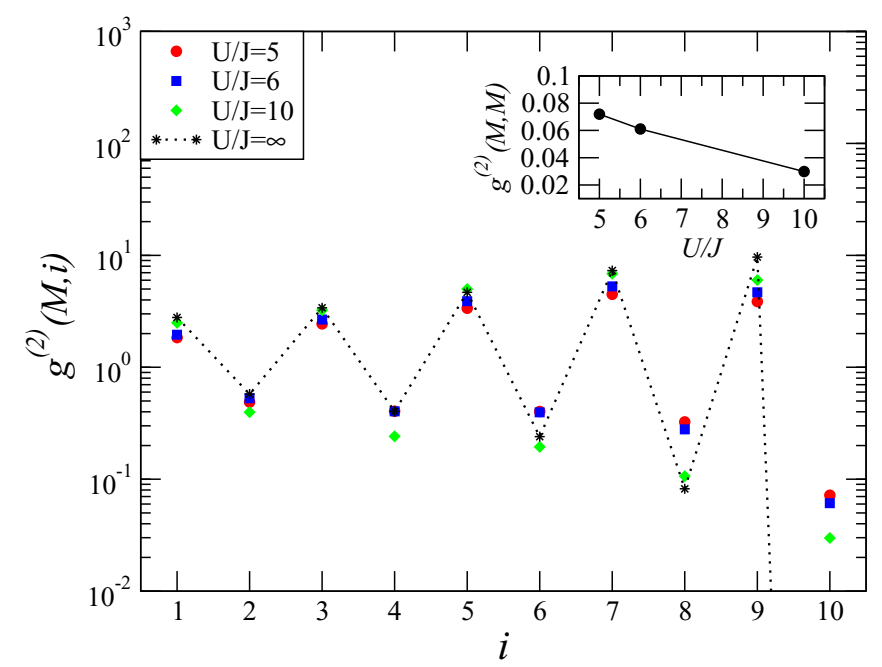

FIG. 9. (Color online) The normalized two-body correlation function on resonance with the $U / J$ dependent two-photon peak (see Fig. 8). Here, the cavity on the opposite side with respect to the driving laser is taken as reference. In the inset, the autocorrelation of the $M$ th cavity is shown as a function of $U / J$. The parameters are set as in Fig. 8.

same probability, but with an amplitude carrying opposite sign. This is due to the fact that when the laser is resonant with the two-photon state $\left|1: k_{p} ; 1: k_{q}\right\rangle$ we get a level scheme similar to Fig. 5 where the state $\left|k_{p}, k_{q}\right\rangle$ should be replaced with the state $\left|1: k_{p} ; 1: k_{q}\right\rangle$ and the dissipation allows (incoherent) transition from the target two-body state $\left|1: k_{p} ; 1: k_{q}\right\rangle$ to $\left|k_{p}\right\rangle$ and $\left|k_{q}\right\rangle$ only. The one-body states have equal and opposite energy $( \pm \Delta)$ with respect to the vacuum and are coupled to $\left|1: k_{p} ; 1: k_{q}\right\rangle$ with a matrix element

$$
\begin{aligned}
& \left\langle k_{n}|\hat{\mathcal{H}}|_{U=0} \mid 1: k_{p} ; 1: k_{q}\right\rangle \\
& \quad=F \sqrt{\frac{2}{M+1}}\left(\delta_{k_{n}, k_{p}} \sin k_{q}+\delta_{k_{n}, k_{q}} \sin k_{p}\right) .
\end{aligned}
$$

For this reason, despite the state $\left|1: k_{5} ; 1: k_{6}\right\rangle$ is an eigenstate of the free Hamiltonian, it is not visible in the spectrum of the total population (see Fig. 8).

\section{B. Interacting case}

Switching on the nonlinearity, the resonances at $\omega_{p}=\mathcal{E}\left(k_{n}\right)$ are split and shifted: the harmonicity of the spectrum is broken and the photon blockade takes place. Specifically, the twophoton state $\left|2: k_{n}\right\rangle$ is no longer an eigenstate of the interacting Hamiltonian. In the weakly interacting limit $(U / J \ll 1)$, the relative resonance is blue-shifted proportionally to $U / M$ at first order in perturbation theory $[12,16]$. Such regime is in principle difficult to study within the MPO approach because of the large dimension of the local Hilbert space, but can be explored by means of perturbation theory in the parameter $U / J$ or using a classical discrete nonlinear Schrödinger equation [7]. For larger, but still finite values of $U / J$, as shown in Fig. 8, the resonance continues to be blue-shifted approaching asymptotically the fermionized value [12]. In this regime, an MPO approach gives reliable results. Interestingly, we found that the needed local dimension for moderate driving strength
$(F / \gamma=2)$ and $M=10$ is just $d=3$ for all the values of $U / J$ that we considered (not shown).

In the top panel of Fig. 8 the spectra of the total number of photons in the NESS $n_{T}=\sum_{i=1}^{M}\left\langle\hat{a}_{i}^{\dagger} \hat{a}_{i}\right\rangle$ is shown. We note that, being the eigenstates of $\mathcal{H}_{0}$ extended independently from $U / J$, it exhibits all the features of the population in the $M$ th cavity (bottom panel).

The degree of nonlinearity of the system also influences the statistics of the output radiation. In Fig. 9, we show the behavior of $g^{(2)}$ for different values of the Kerr nonlinearity $U / J$ (we keep the driving on resonance with the $U / J$ dependent two-photon peak of Fig. 8). The oscillatory behavior of the cross correlations that we found in the TG limit persists also at finite interaction. The autocorrelation of the light in the $M$ th cavity exhibits antibunching $\left[g^{(2)}(M, M)<1\right]$ for all the values of $U / J$ probed (see inset of Fig. 9). Such antibunching is more pronounced as the nonlinearity is increased. For a detailed discussion about how the light statistics is related to the nonlinearity strength in CCAs, see Ref. [17].

\section{v. CONCLUSIONS}

In this work, we studied an array of coupled nonlinear cavities subjected to dissipation and driven, at one end, by a coherent source. By means of numerical simulations and inputoutput formalism we characterized the transport properties analyzing both the populations and the correlations of the transmitted light in the nonequilibrium steady state emerging from the interplay of driving and dissipation.

We found that the formation of strongly correlated (manybody) states of light in the NESS determines the transport properties of the system we consider. Remarkably, strong correlations play a dominant role also in very large arrays (we simulated up to 60 cavities). This, we believe, is a nontrivial observation since the incoherent photon leakage from each cavity is expected to kill coherence between different cavities. Additionally, since we refill the array just from one end (while dissipation occurs extensively), it is not obvious that transport in large systems can take place at all. We found that the transmission of photons through the array displays single-photon and multiphoton peaks, which reveal the level structure of the array resulting from the competition between photon hopping and Kerr-type nonlinearity. For weak driving strength, only single-photon states appear in the transmission spectra. As the pump strength is increased, resonances related to many-body states start to appear. Our work establishes that photon transport is controlled by many-body resonances related to extended states in the cavity array.

In the Tonks-Girardeau limit, the number of transmitted photons on resonance with single-photon and multiphoton states has been fully characterized and the presence of strongly correlated states of light in the steady state manifests in the behavior of the two-body correlation function which signals a perfect antibunched statistics of the output field. In this regime, we developed some truncated effective models (based on a careful identification of the relevant degrees of freedom) which allow us to confirm our numerical results and considerably increase the understanding of the physics of this complex system. The agreement is almost perfect, both for the populations and for the correlation functions, confirming the 
effectiveness of the MPO method for the investigation of large (one-dimensional) open quantum many-body systems.

We went beyond the Tonks-Girardeau limit. We analyzed the case of a finite Kerr nonlinearity. Also in this case we found that the structure of the strongly correlated states in the steady state rules the transport. In particular, moving from the linear regime to the Tonks-Girardeau limit, the harmonic structure of the spectrum is progressively lost, dramatically affecting (and inhibiting) the transport properties of the array. This effect is the generalization to extended systems of the well-known single-cavity photon-blockade phenomena. Quite interesting in this respect would be the analysis of the transport in the opposite regime in which the hopping is dominating over the local nonlinearity. Here, one expects that the spectrum of the Hamiltonian has low-lying soundlike modes leading, in equilibrium, to quasi-long-range order. It would be very interesting to see how photon transmission is modified in this "superfluid" regime. Unfortunately, the case of small Kerr nonlinearity is difficult to handle with the numerical methods used here as the dimension of the local Hilbert space grows enormously. A dissipative Luttinger liquid description or the truncated Wigner methods $[39,40]$ are probably much more appropriate in this regime.

To conclude, our analysis can be viewed as the multicavity generalization of the classic experiments on photon blockade. By exploring these complex architectures that show a manybody photon-blockade effect, as in this paper, an additional ingredient to tune and control photon transmission at singleor few-photon level can be realized. Note that by driving all the cavities, one is limited by symmetry reasons to address only few eigenstates of the system. On the contrary, in our configuration, by suitably adjusting the laser-cavity detuning, it is possible to excite the whole many-body spectrum of the system, paving the way to the possibility to perform a complete system spectroscopy of the array studying transport.

\section{ACKNOWLEDGMENTS}

We would like to thank A. Mari for fruitful discussions. We acknowledge financial support by the ERC through the QGBE grant, by the Autonomous Province of Trento, partly through the project "On silicon chip quantum optics for quantum computing and secure communications" ("SiQuro"), by EU through the grants IP-project SIQS, and the STREP project THERMIQ, by Regione Toscana POR FSE 2007-2013, and by the Italian MIUR through Projects No. RBFR12NLNA and No. 2010LLKJBX006 "Fenomeni quantistici collettivi: dai sistemi fortemente correlati ai simulatori quantistici".

\section{APPENDIX A: EFFECTIVE MODELS}

In this work, we compared the results of the MPO simulations with the outcome of some truncated effective models. All the results of this appendix refer to the TG limit $(U / J=\infty)$. The first model we consider takes into account only the one-particle sector of $\hat{\mathcal{H}}_{0}$ and the vacuum. As in the main text, we will call it the one-body model (OBM). In this model, the vacuum $|0\rangle$ is coupled to the single-particle states $\left|k_{n}\right\rangle$ with a matrix element

$$
F_{k_{n}}=\left\langle 0|\hat{\mathcal{H}}| k_{n}\right\rangle=F \sqrt{\frac{2}{M+1}} \sin k_{n},
$$

where we used $\left|k_{n}\right\rangle=\sqrt{\frac{2}{M+1}} \sum_{i=1}^{M} \sin \left(k_{n} i\right) \hat{a}_{i}^{\dagger}|0\rangle$ with $k_{n}=$ $n \pi /(M+1)$ and $n=1, \ldots, M$ as imposed by the open boundary conditions. As it is clear from Eq. (A1), in this driving scheme, all the single-particle states can be excited. The nonunitary part of the evolution of the master equation (1) is governed by the Lindbladian term (7). In this case, it is more convenient to work with the annihilation and creation operators of Bloch modes which are related the photons annihilation and creation operators as usual $\hat{b}_{k_{n}}=$ $\sqrt{2 /(M+1)} \sum_{i=1}^{M} \sin \left(k_{n} i\right) \hat{a}_{i}$. Using the orthogonality relation $\frac{2}{M+1} \sum_{l=1}^{M} \sin \left(k_{p} l\right) \sin \left(k_{q} l\right)=\delta_{k_{p}, k_{q}}$, it is easy to show that

$$
\begin{aligned}
\mathcal{L}[\rho] & =\frac{\gamma}{2} \sum_{i=1}^{M}\left(2 \hat{a}_{i} \rho \hat{a}_{i}^{\dagger}-\hat{a}_{i}^{\dagger} \hat{a}_{i} \rho-\rho \hat{a}_{i}^{\dagger} \hat{a}_{i}\right) \\
& =\frac{\gamma}{2} \sum_{n=1}^{M}\left(2 \hat{b}_{k_{n}} \rho \hat{b}_{k_{n}}^{\dagger}-\hat{b}_{k_{n}}^{\dagger} \hat{b}_{k_{n}} \rho-\rho \hat{b}_{k_{n}}^{\dagger} \hat{b}_{k_{n}}\right)
\end{aligned}
$$

Also in this case all we need are the matrix elements $\left\langle 0\left|\hat{b}_{k_{m}}\right| k_{n}\right\rangle=\left\langle 0\left|\hat{b}_{k_{m}} \hat{b}_{k_{n}}^{\dagger}\right| 0\right\rangle=\delta_{k_{m}, k_{n}}$. The result explains why we worked in this different basis for the Lindbladian term. The OBM is expected to work when the driving is weak $(F / \gamma \ll 1)$ and therefore only the single-particle states play a role in the dynamics. Nevertheless, if the pump is not resonant with a two-photon state so that the excitation of many-body states is strongly inhibited, the OBM is expected to work even if the driving is not weak (see, e.g., Figs. 3 and 4). Of course, any kind of effective model one could think will fail for large driving strength $(F / \gamma \gg 1)$ because at a certain point the excitation of the states not included in the model starts to be not negligible. A clear example is given in Fig. 3. When the laser is resonant with a one-photon state $\left|k_{n}\right\rangle$ and the others resonances are well separated in energy with respect to their width, we can further simplify the model taking into account the target state and the vacuum only. We will refer to this model as the two-level model (TLM). The TLM can be solved analytically for the steady state. What we get is

$$
\begin{aligned}
\left\langle k_{n}\left|\rho^{\mathrm{NESS}}\right| k_{n}\right\rangle & =\frac{F_{k_{n}}^{2}}{2 F_{k_{n}}^{2}+(\gamma / 2)^{2}}, \\
\left\langle k_{n}\left|\rho^{\mathrm{NESS}}\right| 0\right\rangle & =i \frac{2 F_{k_{n}}}{\gamma}\left(\frac{2 F_{k_{n}}^{2}}{2 F_{k_{n}}^{2}+(\gamma / 2)^{2}}-1\right),
\end{aligned}
$$

where $\rho^{\text {NESS }}$ is the NESS density matrix.

When the laser is resonant with a two-photon state $\left|k_{p}, k_{q}\right\rangle$, we used another effective model which takes into account all the single-particle states $\left|k_{n}\right\rangle$, the target two-body state $\left|k_{p}, k_{q}\right\rangle$, and the vacuum $|0\rangle$. We will refer to this model as the two-body model (TBM). The vacuum is coupled to the single-particle 


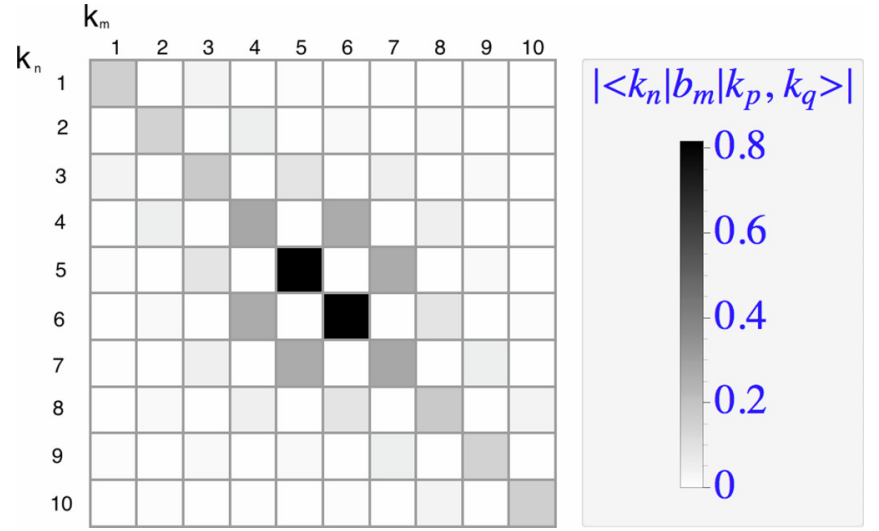

FIG. 10. (Color online) The absolute value of the matrix element $\left\langle k_{n}\left|\hat{b}_{m}\right| k_{p}, k_{q}\right\rangle$ for the target two-photon state $\left|k_{5}, k_{6}\right\rangle$ as a function of $k_{n}$ and $k_{m}$ for $M=10$.

states as before, while the two-body state is coupled to the one-body states with

$$
\begin{aligned}
A_{k_{n}, k_{p}, k_{q}} & =\left\langle k_{n}|\hat{\mathcal{H}}| k_{p}, k_{q}\right\rangle \\
& =F \sqrt{\frac{2}{M+1}}\left[\delta_{k_{n}, k_{p}} \sin k_{q}-\delta_{k_{n}, k_{q}} \sin k_{p}\right] .
\end{aligned}
$$

Here, we explicitly used the structure of the two-body eigenstates of $\mathcal{H}_{0}, \quad$ i.e., $\left|k_{p}, k_{q}\right\rangle=$ $\frac{1}{M+1} \sum_{i, j=1}^{M} f_{k_{p}, k_{q}}(i, j) \quad \hat{a}_{i}^{\dagger} \hat{a}_{j}^{\dagger}|0\rangle \quad$ where $\quad f_{k_{p}, k_{q}}(i, j)=$ $\operatorname{sgn}(i-j)\left[\sin \left(k_{p} i\right) \sin \left(k_{q} j\right)-\sin \left(k_{p} j\right) \sin \left(k_{q} i\right)\right]$ and the orthogonality relation $\frac{2}{M+1} \sum_{l=1}^{M} \sin \left(k_{p} l\right) \sin \left(k_{q} l\right)=\delta_{k_{p}, k_{q}}$. Remarkably the state $\left|k_{p}, k_{q}\right\rangle$ is coupled only with the states $\left|k_{p}\right\rangle$ and $\left|k_{q}\right\rangle$. This is unexpected because the state $\left|k_{q}, k_{q}\right\rangle$ has a very complicated structure in the momentum space. Nevertheless, shining only the first cavity (or equivalently the last one) we obtain a result very similar to the free case where

$$
\begin{aligned}
& \left\langle k_{n}|\hat{\mathcal{H}}|_{U=0} \mid 1: k_{p} ; 1: k_{q}\right\rangle \\
& \quad=F \sqrt{\frac{2}{M+1}}\left[\delta_{k_{n}, k_{p}} \sin k_{q}+\delta_{k_{n}, k_{q}} \sin k_{p}\right] .
\end{aligned}
$$

Analogously, it can be shown that the three-photon state $\left|k_{a}, k_{b}, k_{c}\right\rangle$ is coupled only with the states $\left|k_{a}, k_{b}\right\rangle,\left|k_{b}, k_{c}\right\rangle$, and $\left|k_{a}, k_{c}\right\rangle$.

For the dissipative part of the evolution, additionally to the matrix elements evaluated above, we need to compute

$$
\begin{aligned}
\left\langle k_{n}\left|\hat{b}_{m}\right| k_{p}, k_{q}\right\rangle= & \left(\frac{2}{M+1}\right)^{2} \frac{1}{2} \sum_{i, j, l, s=1}^{M} \sin \left(k_{n} l\right) \sin \left(k_{m} s\right) \\
& \times f_{k_{p}, k_{q}}(i, j)\left\langle 0\left|\hat{a}_{l} \hat{a}_{s} \hat{a}_{i}^{\dagger} \hat{a}_{j}^{\dagger}\right| 0\right\rangle \\
= & \left(\frac{2}{M+1}\right)^{2} \sum_{i, j=1}^{M} \sin \left(k_{n} i\right) \sin \left(k_{m} j\right) f_{k_{p}, k_{q}}(i, j) .
\end{aligned}
$$

Expression (A6) cannot be simplified further. It keeps track of the very rich distribution of $\left|k_{p}, k_{q}\right\rangle$ in the momentum space. As it is shown in Fig. 10, in contrast to the Hamiltonian evolution, the dissipative dynamics couples incoherently the two-photon

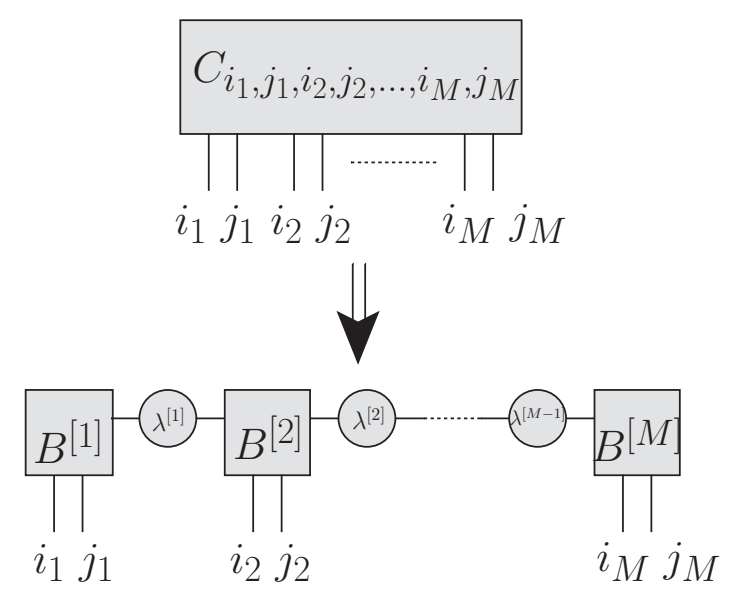

FIG. 11. Graphical representation of the density matrix in a tensor-network language. Each block is a multi-index tensor, open links represent the free indexes, while connected links stand for the contracted indexes.

state $\left|k_{p}, k_{q}\right\rangle$ not only to $\left|k_{p}\right\rangle$ and $\left|k_{q}\right\rangle$. The typical level scheme is shown in Fig. 5.

\section{APPENDIX B: REMARKS ON THE MATRIX-PRODUCT-OPERATOR APPROACH}

This summary about the superoperator renormalization group technique is based on the appendix given in Ref. [41]. For the time evolution toward the NESS of the density matrix we exploit an algorithm based on the time-evolving block decimation (TEBD) scheme [37,38] extended to the open systems $[34,35]$. In this framework the density matrix for an

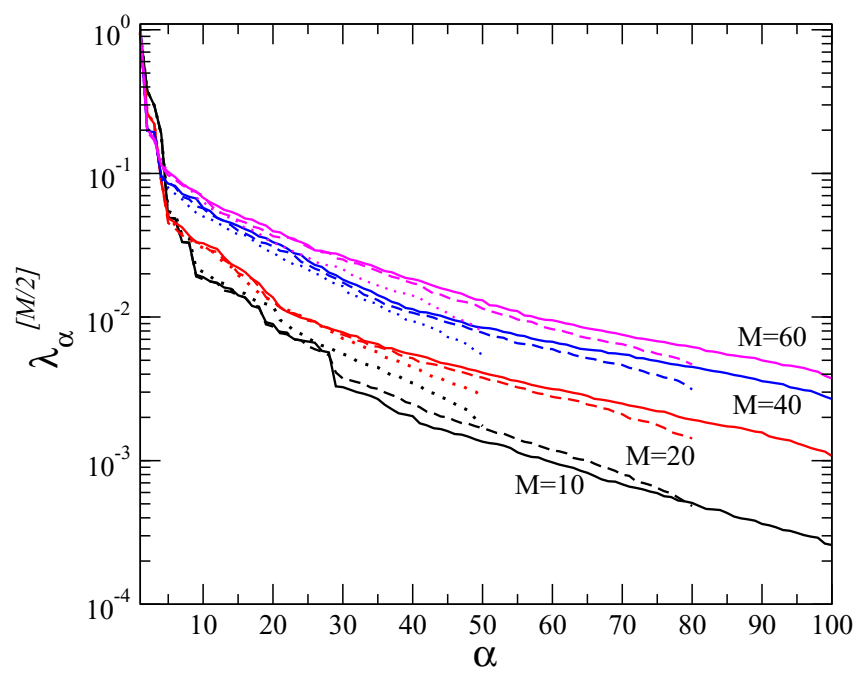

FIG. 12. (Color online) The spectrum of the Schmidt coefficients $\lambda_{\alpha}^{[M / 2]}$ for a symmetric bipartition. We consider various system sizes and different bond-link dimensions: $\chi=50$ (dotted lines), 80 (dashed lines), 100 (continuous lines). The laser is resonant with the single-particle state $\left|k_{M / 2}\right\rangle$ and the parameters are $U / J=\infty, J / \gamma=$ $20, \omega_{0} / \gamma=1$, and $F / \gamma=1$. 


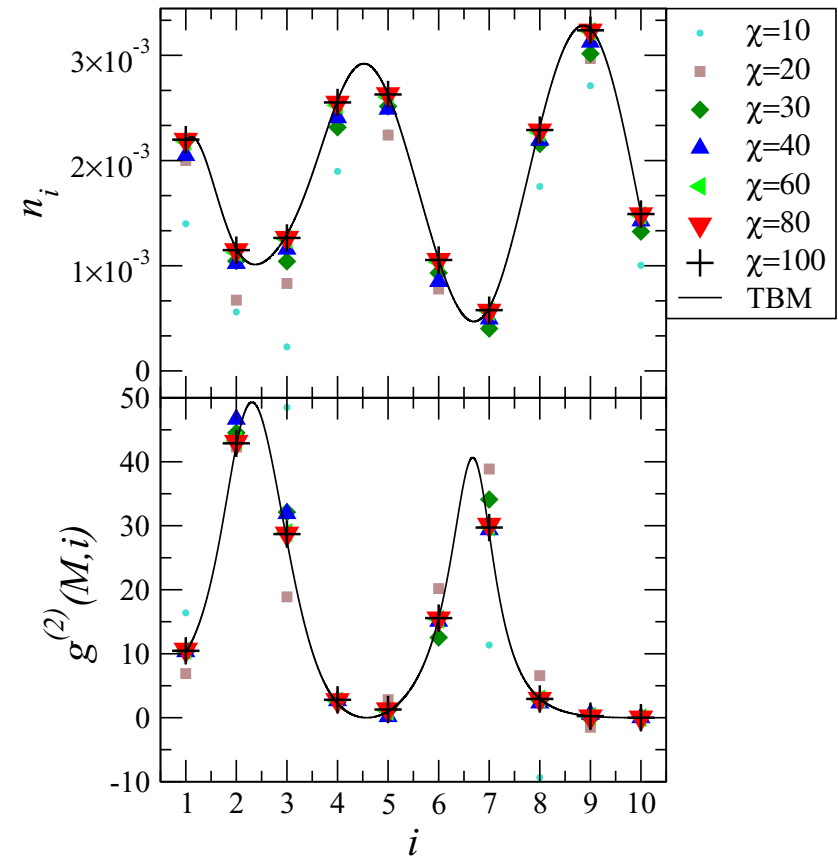

FIG. 13. (Color online) Local density (top panel) and normalized two-body function (lower panel) for different values of the bondlink dimension $\chi$. As indicated in the legend, the solid lines are the predictions of the TBM (see Appendix A). Here, the laser is resonant with the two-photon state $\left|k_{2}, k_{3}\right\rangle$ of an array of $M=10$ cavities. The parameters are $U / J=\infty, J / \gamma=20, \omega_{0} / \gamma=1$, and $F / \gamma=1$. The data for $\chi=100$ are shown in the left lower panel of Fig. 6 (local density) and in the right lower panel of Fig. 7 (two-body function).

array of $M$ cavities and open boundary conditions

$$
\left.\left.\rho=\sum_{i_{\alpha}, j_{\alpha}=1}^{d} C_{i_{1} \ldots i_{L}, j_{1} \ldots j_{M}} \| i_{1} \ldots i_{M}, j_{1} \ldots j_{M}\right\rangle\right\rangle
$$

is written as a MPS in the enlarged Hilbert space of dimension $d^{2}$, where $d$ is the dimension of the local Hilbert space $\mathbb{H}$. In the specifics, a repeated application of singular value decompositions of the tensor $C_{i_{1} \ldots i_{L}, j_{1} \ldots j_{M}}$ leads to the following representation (see Fig. 11):

$$
\begin{aligned}
\rho= & \sum_{i_{\alpha}, j_{\alpha}=1}^{d} \sum_{\alpha, \beta, \ldots, \gamma=1}^{\chi} B_{1, \alpha}^{[1] i_{1}, j_{1}} \lambda_{\alpha}^{[1]} B_{\alpha, \beta}^{[2] i_{2}, j_{2}} \lambda_{\beta}^{[2]} \\
& \left.\left.\times \ldots \lambda_{\gamma}^{[M-1]} B_{\gamma, 1}^{[M] i_{M}, j_{M}} \| i_{1} \ldots i_{M}, j_{1} \ldots j_{M}\right\rangle\right\rangle .
\end{aligned}
$$

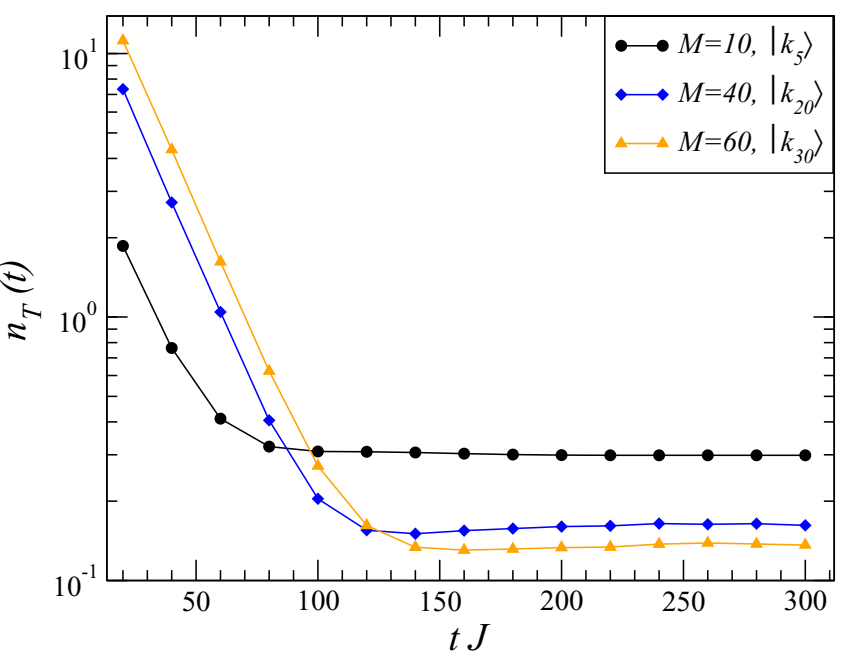

FIG. 14. (Color online) Evolution toward the NESS value of the total population for different system sizes and target states, as indicated in the legend. The initial state is random and the parameters are set as in Fig. 12.

Here, $\left.\left.\| i_{1} \ldots i_{M}, j_{1} \ldots j_{M}\right\rangle\right\rangle=\bigotimes_{a=1}^{M}\left|i_{a}\right\rangle\left\langle j_{a}\right|$ represents a basis for the density matrix in the product Hilbert space $\mathbb{H}^{\otimes M} \otimes \mathbb{H}^{\otimes M}$.

As explained in Ref. [34], if the Schmidt spectrum $\lambda_{\alpha}^{[i]}$ decays fast enough, it can be truncated keeping only the $\chi$ largest Schmidt values. In our simulations, we fix $\chi=100$. This choice is well justified, on the basis of the behavior of the Schmidt spectrum for a typical choice of parameters (see Fig. 12). This is reflected on how the observables studied in this work (local density and two-body function) converge as $\chi$ is increased for a typical choice of parameters (see Fig. 13). As an extension of the entanglement entropy, the operator space entanglement entropy $[42,43]$ of a bipartition $A$ of size $l$ is straightforwardly related to the behavior of the Schmidt spectrum:

$$
\mathcal{S}_{l}=-2 \sum_{\alpha}\left(\lambda_{\alpha}^{[l]}\right)^{2} \log _{2} \lambda_{\alpha}^{[l]} .
$$

The time evolution is then performed using a Suzuki-Trotter decomposition (at fourth order in our simulations) of timeevolution superoperator. Once the NESS is reached, the expectation values of some operators are obtained in the standard way $\langle\hat{O}\rangle=\mathcal{Z}^{-1} \operatorname{Tr}\left[\rho^{\text {NESS }} \hat{O}\right]$ where $\mathcal{Z}=\operatorname{Tr} \rho^{\text {NESS }}$ is the partition function. The typical evolution toward the NESS value for the observable $n_{T}=\sum_{i=1}^{M}\left\langle\hat{a}_{i}^{\dagger} \hat{a}_{i}\right\rangle$ is shown in Fig. 14 for different system sizes.
[1] M. J. Hartmann, F. G. S. L. Brandão, and M. B. Plenio, Nat. Phys. 2, 849 (2006).

[2] A. D. Greentree, C. Tahan, J. H. Cole, and L. C. L. Hollenberg, Nat. Phys. 2, 856 (2006).

[3] D. G. Angelakis, M. F. Santos, and S. Bose, Phys. Rev. A 76, 031805(R) (2007).
[4] M. J. Hartmann, F. G. S. L. Brandão, and M. B. Plenio, Laser Photon. Rev. 2, 527 (2008).

[5] A. Tomadin and R. Fazio, J. Opt. Soc. Am. 27, A130 (2010).

[6] A. A. Houck, H. E. Türeci, and J. Koch, Nat. Phys. 8, 292 (2012).

[7] I. Carusotto and C. Ciuti, Rev. Mod. Phys. 85, 299 (2013). 
[8] D. L. Underwood, W. E. Shanks, J. Koch, and A. A. Houck, Phys. Rev. A 86, 023837 (2012).

[9] M. Abbarchi, A. Amo, V. G. Sala, D. D. Solnyshkov, H. Flayac, L. Ferrier, I. Sagnes, E. Galopin, A. Lemaître, G. Malpuech, and J. Bloch, Nat. Phys. 9, 275 (2013).

[10] K. Toyoda, Y. Matsuno, A. Noguchi, S. Haze, and S. Urabe, Phys. Rev. Lett. 111, 160501 (2013).

[11] D. Rossini and R. Fazio, Phys. Rev. Lett. 99, 186401 (2007).

[12] I. Carusotto, D. Gerace, H. E. Türeci, S. De Liberato, C. Ciuti, and A. Imamoğlu, Phys. Rev. Lett. 103, 033601 (2009).

[13] A. Tomadin, V. Giovannetti, R. Fazio, D. Gerace, I. Carusotto, H. E. Türeci, and A. Imamoğlu, Phys. Rev. A 81, 061801(R) (2010).

[14] M. J. Hartmann, Phys. Rev. Lett. 104, 113601 (2010).

[15] F. Nissen, S. Schmidt, M. Biondi, G. Blatter, H. E. Türeci, and J. Keeling, Phys. Rev. Lett. 108, 233603 (2012).

[16] T. Grujic, S. R. Clark, D. G. Angelakis, and D. Jaksch, New J. Phys. 14, 103025 (2012).

[17] T. Grujic, S. R. Clark, D. Jaksch, and D. G. Angelakis, Phys. Rev. A 87, 053846 (2013).

[18] J. Jin, D. Rossini, R. Fazio, M. Leib, and M. J. Hartmann, Phys. Rev. Lett. 110, 163605 (2013); J. Jin, D. Rossini, M. Leib, M. J. Hartmann, and R. Fazio, Phys. Rev. A 90, 023827 (2014).

[19] T. Yuge, K. Kamide, M. Yamaguchi, and T. Ogawa, J. Phys. Soc. Jpn. 83, 123001 (2014).

[20] J. Ruiz-Rivas, E. del Valle, C. Gies, P. Gartner, and M. J. Hartmann, Phys. Rev. A 90, 033808 (2014).

[21] J. T. Shen and S. Fan, Phys. Rev. Lett. 95, 213001 (2005); 98, 153003 (2007); Phys. Rev. A 79, 023837 (2009); 79, 023838 (2009).

[22] D. E. Chang, A. S. Sorensen, E. Demler, and M. D. Lukin, Nat. Phys. 3, 807 (2007).
[23] P. Longo, P. Schmitteckert, and K. Busch, Phys. Rev. Lett. 104, 023602 (2010); Phys. Rev. A 83, 063828 (2011).

[24] S. Felicetti, G. Romero, D. Rossini, R. Fazio, and E. Solano, Phys. Rev. A 89, 013853 (2014).

[25] M. Hafezi, D. E. Chang, V. Gritsev, E. Demler, and M. D. Lukin, Europhys. Lett. 94, 54006 (2011).

[26] J.-Q. Liao, Z. R. Gong, L. Zhou, Y.-X. Liu, C. P. Sun, and F. Nori, Phys. Rev. A 81, 042304 (2010).

[27] A. Imamoğlu, H. Schmidt, G. Woods, and M. Deutsch, Phys. Rev. Lett. 79, 1467 (1997).

[28] S. Rebić et al., J. Opt. B: Quantum Semiclass. Opt. 1, 490 (1999).

[29] J. Kim et al., Nature (London) 397, 500 (1999).

[30] K. M. Birnbaum et al., Nature (London) 436, 87 (2005).

[31] T. C. H. Liew and V. Savona, Phys. Rev. Lett. 104, 183601 (2010).

[32] V. S. Ferretti, V. Savona, and D. Gerace, New J. Phys. 15, 025012 (2013).

[33] M. Girardeau, J. Math. Phys. 1, 516 (1960).

[34] M. Zwolak and G. Vidal, Phys. Rev. Lett. 93, 207205 (2004).

[35] F. Verstraete, J. J. Garcia-Ripoll, and J. I. Cirac, Phys. Rev. Lett. 93, 207204 (2004).

[36] D. F. Walls and G. J. Milburn, Quantum Optics (Springer, Berlin, 2008).

[37] G. Vidal, Phys. Rev. Lett. 91, 147902 (2003).

[38] G. Vidal, Phys. Rev. Lett. 93, 040502 (2004).

[39] A. Sinatra, C. Lobo, and Y. Castin, J. Phys. B: At., Mol. Opt. Phys. 35, 3599 (2002).

[40] I. Carusotto and C. Ciuti, Phys. Rev. B 72, 125335 (2005).

[41] L. Bonnes, D. Charrier, and A. M. Läuchli, Phys. Rev. A 90, 033612 (2014).

[42] T. Prosen and I. Pižorn, Phys. Rev. A 76, 032316 (2007).

[43] M. Žnidarič, T. Prosen, and I. Pižorn, Phys. Rev. A 78, 022103 (2008). 